\title{
Análisis de Factores que afectan la Caracterización de las Propiedades del Carbón*
}

\section{Analysis of Influential Factors in Coal Properties Characterization}

\author{
DOI: http://dx.doi.org/10.17981/ingecuc.12.1.2016.12 \\ Artículo de Investigación Científica - Fecha de Recepción: 27 de Agosto de 2015 - Fecha de Aceptación: 18 de Diciembre de 2015 \\ Natalia Cifuentes Baquero \\ Ingeniero Industrial. Universidad Escuela Colombiana de Ingeniería Julio Garavito. Bogotá (Colombia). \\ natalia.cifuentes@mail.escuelaing.edu.co \\ Diana Carolina Franco Sánchez \\ Ingeniero Industrial. Universidad. Escuela Colombiana de Ingeniería Julio Garavito. Bogotá (Colombia). \\ diana.franco-s@mail.escuelaing.edu.co \\ Andrés Meleg Ruttkay \\ M.Sc en Diseño de Máquinas. Universidad Escuela Colombiana de Ingeniería Julio Garavito. Bogotá (Colombia). \\ andresmelegr@gmail.com \\ Para citar este artículo / To reference this paper: \\ N. Cifuentes Baquero, D. C. Franco Sánchez y A. Meleg Ruttkay, "Análisis de factores que afectan la caracterización \\ de las propiedades del carbón”, INGE CUC, vol. 12, no. 1, pp. 107-119, 2016. DOI: http://dx.doi.org/10.17981/ \\ ingecuc.12.1.2016.12
}

\begin{abstract}
Resumen--En este trabajo se estudia el problema relacionado con la variación de las propiedades del carbón bituminoso, utilizado en una planta de energía eléctrica colombiana. La estrategia implementada fue la metodología de diseño de experimentos (DOE) para encontrar las causas de estas variaciones, cambiando las condiciones de almacenamiento, afectadas por el clima y las diferentes formas de manejo de muestras. El estudio contempla el diseño, desarrollo y análisis de tres experimentos con el fin de identificar la influencia de parámetros como el tiempo y utilización del carbón almacenado, el clima antes del consumo y algunos procedimientos de muestreo y de esta forma explicar la divergencia entre las propiedades del carbón medidas en el patio y durante la trituración justo antes de ser quemado. Los resultados mostraron que el tiempo de almacenamiento en el patio y el desempeño del sistema automático de muestreo son la causa principal del problema.
\end{abstract}

Palabras Claves-- Carbón Bituminoso; Poder Calorífico; Análisis Último; Muestreo de Carbón; Generación de Energía; Análisis de Datos.

\begin{abstract}
This paper studies the problem related to the variation of measured properties of a bituminous coal, fired in a Colombian electric power plant. The strategy was to implement a DOE methodology to find the causes of these variations, changing the storage conditions and relating it to the weather and different ways of handling the samples. The study contemplates the design, deployment and analysis of three experiments in order to trace the influence of such parameters as time and deplete of storage, weather evolution prior to consumption and some sampling procedures and in this way explain the divergence of coal properties between the reception in the yards and its medium grinding just before firing. The results had shown that stocking time in yards and the automatic system's performance, as the main cause of the problem.
\end{abstract}

Keywords-- Bituminous Coal; Heating Value; Ultimate Analysis; Coal Sampling; Power Generation; Data Analysis.

\footnotetext{
"Artículo de investigación científica derivado del proyecto de investigación titulado: "Diseño de experimentos aplicado al muestreo de carbón utilizado en la producción de energía eléctrica.”. Financiado por la Escuela Colombiana de Ingeniería Julio Garavito y la Compañia Eléctrica de Sochagota (CES). Fecha de inicio: enero de 2014. Fecha de finalización: junio de 2015.
} 


\section{INTRODUCCIÓN}

En el negocio de producción de energía eléctrica la generación térmica se ha ido posicionando como la principal alternativa para garantizar la demanda energética de un entorno. En Colombia las centrales termoeléctricas han ido incrementando su capacidad y por ende sus procesos internos son cada día más regulados para hacer de este un negocio rentable.

La Central Termoeléctrica objeto de estudio es una planta colombiana de generación de energía eléctrica que opera como "Stand By" y cuya producción utiliza como único combustible carbón bituminoso pulverizado [1]. El consumo mensual de material es de aproximadamente 45.000 toneladas (Ton.) y tiene una capacidad instalada de $150 \mathrm{Me}-$ gaWatts (MW).

A petición de la Central Termoeléctrica la información correspondiente a las propiedades del carbón presentada en las tablas y gráficas de este documento fue modificada, de tal manera que se visualiza el comportamiento de las variables, sin embargo se mantiene la confidencialidad de la información suministrada.

En el cumplimiento de las metas de generación están involucrados dos factores que afectan el desempeño del proceso: la eficiencia de la caldera y la calidad del carbón utilizado. A pesar de los controles que la Central Termoeléctrica efectúa sobre la eficiencia de la caldera, cuyo valor ha sido establecido previamente y el control de la calidad del carbón comprado a los diferentes proveedores, existen inconsistencias entre la cantidad de MW que se espera generar y los MW obtenidos tras el proceso de combustión del carbón (KW-h). Los valores de la calidad del carbón son obtenidos a partir del estudio de las propiedades físicas y químicas, para lo se ejecuta un muestreo y posterior análisis de laboratorio, con el fin de verificar que el material entregado por el proveedor cumple con el porcentaje de ceniza máximo pactado (20\%), además de obtener el parámetro para calcular el pago que se debe hacer al proveedor, dado que es el poder calorífico del material la principal característica para establecer la prima o sanción aplicadas para calcular el precio pagado.

La diferencia positiva existente entre las propiedades medidas en la pila de almacenamiento y posteriormente durante el consumo, resulta en un déficit económico evidenciado en los análisis financieros de la compañía. Con el fin de estudiar los factores que afectan la calidad de carbón utilizado para la generación y el desajuste en las mediciones efectuadas, se estableció un proyecto de investigación basado en el análisis de las propiedades físicas del material, que permitiera identificar las causas de dicha variación.
El enfoque del análisis se establece después de estudiar la cadena del carbón, desde que se extrae de la mina hasta que es quemado para producir energía. En este proceso se tienen en cuenta las condiciones de manejo durante las etapas de transporte y almacenamiento que puedan causar cambios en sus propiedades debido a las condiciones ambientales bajo las que es manejado [2].

Dados los escenarios de transporte y almacenamiento establecidos en la Central Termoeléctrica, en este trabajo se estudian específicamente las condiciones y procedimientos de muestreo utilizados [3], con el fin de verificar la representatividad de las muestras analizadas [4] e identificar los factores de la operación diaria que puedan alterar las propiedades medidas en el carbón.

\section{Oвjetivos}

\section{A. Objetivo especifico}

Identificar las causas de la variación del valor del poder calorífico del carbón medido en las muestras tomadas durante la recepción, el almacenamiento y el consumo del material.

\section{B. Objetivo especifico}

Desarrollar un análisis estadístico de la información suministrada por la Central Termoeléctrica que permita conocer el comportamiento de las propiedades físicas del carbón, en los diferentes escenarios de medición, para establecer las relaciones entre propiedades y como estas afectan los valores del poder calorífico del carbón en las diferentes etapas del proceso.

\section{III.Metodología}

\section{A. Construcción del plan experimental}

Para iniciar esta investigación fue necesario estudiar el manejo del carbón dentro de la Termoeléctrica y de esta manera establecer algunos factores preliminares sobre los que era posible iniciar el estudio. Se analizaron las dos formas de muestreo desarrolladas para hacer la medición de propiedades y los procedimientos para ejecutar cada uno de ellos. Inicialmente a la llegada del carbón se mide el porcentaje de ceniza, para hacer una evaluación previa de la calidad del mismo, cuando es llevado a la pila de almacenamiento se hace un muestreo manual siguiendo los procedimientos de la norma técnica [5] y finalmente a través de un sistema de banda transportadora se recolecta una muestra con ayuda de un sistema mecánico de muestreo.

El almacenamiento del carbón en el patio se lleva a cabo por medio de dos pilas, una para el 
descargue de material y la otra para el consumo. El tiempo de almacenamiento del material depende del nivel de generación y del inventario mínimo estratégico establecido por la Central Termoeléctrica.

Posterior a la definición de las características del proceso se estudian los estándares de medición de las propiedades del carbón documentados en las normas ASTM (cuyo listado completo se encuentra en la bibliografía). Durante esta revisión se comparan los procedimientos utilizados por La Termoeléctrica con los descritos en las normas técnicas, donde se determinan los métodos para la realización del análisis último del material [6]. Adicionalmente se revisa la operación de otras Termoeléctricas con el fin de encontrar algunos estudios de caso sobre la medición del poder calorífico del carbón [7] y los sistemas de almacenamiento utilizados.

La pérdida de poder calorífico del carbón es atribuida a las condiciones ambientales bajo las que se almacena el material, lo que puede causar oxidación, ganancia de humedad o perdida de partículas [2], sin embargo el enfoque de la revisión bibliográfica fue el análisis de los procedimientos bajo los cuales se lleva a cabo la recolección de muestras [5] y las condiciones de almacenamiento en un patio de carbón. En las normas ASTM estudiadas para conocer los procesos de recolección de muestras de carbón [5] y posterior análisis de laboratorio para conocer las propiedades [8], [9], [10], [11], se examinan los procedimientos utilizados por las centrales térmicas para hacer el muestreo durante la etapa de transporte (recepción), en la pila de almacenamiento y finalmente en la línea de consumo.

Finalmente se planteó un Diseño de Experimentos (DOE) que busca relacionar el cambio en las propiedades, con factores como las características de construcción de la pila de almacenamiento, (posición del material en la pila, exposición al ambiente y las condiciones climáticas) presentadas durante la construcción, almacenamiento y consumo de la pila, las mezclas de carbón de diferentes proveedores y el número de muestras recolectadas para analizar las propiedades. Con este planteamiento se busca establecer el efecto de estos factores y diseñar un procedimiento de muestreo que considere las condiciones que significativamente afectan los resultados medidos.

En la fase inicial del DOE se definieron los objetivos y especificaciones para la realización de tres pruebas preliminares que consideraban procedimientos de muestreo especiales con el fin de evaluar el efecto de las condiciones ambientales y de almacenamiento en los resultados obtenidos. Las condiciones evaluadas en esta fase fueron: trituración del material para el paso hacia la caldera, posición del carbón en la pila de almacenamiento (compactación) y el análisis de material proveniente de un único proveedor.
La primera prueba planteada buscaba encontrar posibles diferencias para una cantidad base de 16 toneladas, entre las muestras tomadas en el patio y las tomadas en la banda, con una diferencia temporal menor a una hora. Adicionalmente se garantizó que el único material a través de la banda correspondía a la cantidad de material mencionada. En los dos escenarios se tomaron muestras de $8 \mathrm{Kg}$ que posteriormente fueron preparadas y analizadas siguiendo los mismos procedimientos [12].

En la segunda prueba se compararon los valores de poder calorífico medidos en el patio y en la línea de consumo, sobre una pila de 45.000 t, a la que además se le tomaron muestras a tres alturas diferentes para evaluar el efecto de la compactación. Las muestras tomadas correspondían a tres puntos de la pila (alto, medio, bajo) y fueron tomadas en 7 fechas específicas, con lo cual se obtuvieron 21 análisis midiendo condiciones especiales.

Finalmente en la tercera prueba, con el fin de evaluar el efecto de las condiciones climáticas, el envejecimiento del material (tiempo de almacenamiento) y las mezclas de carbón de varios proveedores, se solicitó construir una pila de 1500 t provenientes de un único proveedor, construida y almacenada a condiciones ambientales conocidas (temperatura, lluvia y velocidad del viento) y para la que se tomaron muestras siguiendo el mismo patrón de la primera prueba con el fin de validar los resultados obtenidos en esta.

Estas pruebas fueron ejecutadas por la Central Termoeléctrica en sus propios laboratorios, proporcionando todos los recursos físicos para garantizar las condiciones especiales propuestas en la recolección de muestras y los estándares utilizados en los procedimientos de análisis.

Los resultados obtenidos en los análisis consideran el efecto de la humedad en las demás propiedades medidas para el carbón por lo cual se genera el reporte en base como se recibe, como se analiza y base seca, esta última luego de someter el material a un proceso de secado para eliminar la humedad superficial (ver Figura 1). Para el análisis de datos, en este trabajo se toman los resultados en base seca, lo que permite tener en cuenta la correlación existente entre el poder calorífico y el porcentaje de ceniza del material.

\section{B. Análisis de información}

Para realizar el análisis de información fue necesario considerar diferentes escenarios para la recolección de la muestra, los tiempos de almacenamiento de las muestras y la base de referencia para expresar los resultados de la caracterización del carbón. Cada una de estas condiciones genera cambios en las comparaciones planteadas entre los experimentos. Para ejecutar los análisis estadísticos y comparaciones pertinentes, fue necesario realizar unificación de los criterios antes mencionados. 
Sobre el carbón, una vez dentro de la Central Termoeléctrica y antes de ser consumido, se ejecutan pruebas a la entrada de los camiones, en la pila para condiciones especiales de análisis y en la línea de alimentación previa al consumo.

El almacenamiento de muestras se hace a aquellas que son recolectadas en los camiones hasta completar muestras que representan $500 \mathrm{t}$ de material recibido. Este almacenamiento se lleva a cabo garantizando que no existe perdida de humedad en el tiempo que trascurre entre la recolección de la muestra y la preparación de la misma para ser analizada, este tiempo es aleatorio de acuerdo a la frecuencia y cantidad recibida de material. Para efectos de análisis se asume que no existe perdida de humedad y se realizan las comparaciones con el porcentaje de humedad total encontrado en la muestra, es decir la humedad superficial del material que gana o pierde por condiciones de temperatura y clima y la humedad residual del material.

La base en la que la Central Termoeléctrica unificó el reporte de la información fue "como se recibe", sin embargo estos datos fueron convertidos a base seca de acuerdo a la norma ASTM [13], dada la importancia de estudiar la correlación entre la ceniza y el poder calorífico del material, la cual no es visible cuando el análisis estadístico de la información se ejecuta con información reportada en base como se recibe.

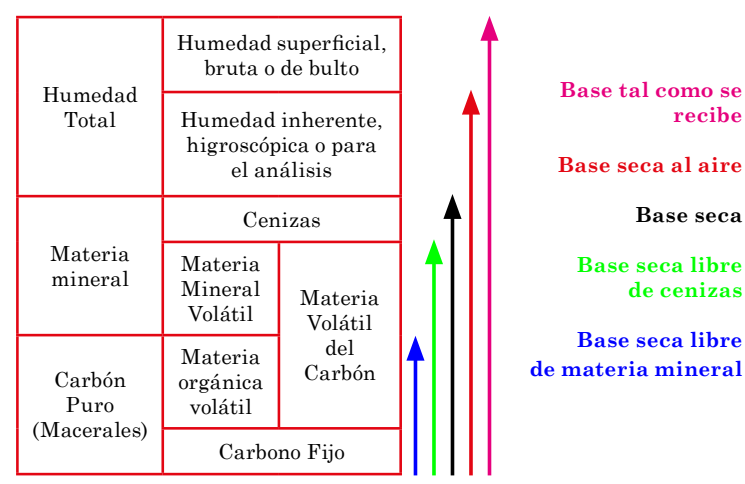

Figura 1. Base de referencia de resultados para la caracterización de carbón Fuente: [14].

ReAlización de los Experimentos

\section{A. Experimento D 1.1 Plan de muestreo preliminar}

Análisis de la variación de propiedades entre los escenarios de medición. El experimento D1.1 busca encontrar la relación entre las propiedades medidas en una muestra tomada en la pila y una muestra tomada durante el consumo. Las condiciones especiales para la recolección de estas muestras es la continuidad con la que son recolectadas en los diferentes escenarios y la evaluación sobre una porción específica de carbón. El procedimiento para la realización de esta prueba fue retirar 16 toneladas de material de una pila existente y recolectar las muestras según el procedimiento que describe la norma [5]. Está cantidad de carbón se deposita en el sistema de alimentación del proceso, una vez se ha retirado de la línea de abastecimiento cualquier otro tipo de carbón. Posteriormente se realiza el muestreo en la banda que transporta el carbón a la caldera para establecer una comparación entre las propiedades medidas en los dos escenarios.

Considerando las condiciones especiales para la realización de la prueba, muestra segmentada y aislada de cualquier otro carbón, se espera encontrar resultados idénticos para las propiedades analizadas en las muestras recolectadas tanto en el patio de carbón como en el consumo posterior al proceso de trituración del material.

La información obtenida en esta prueba indica que el poder calorífico, el porcentaje de humedad y la ceniza del carbón presentan un mejor resultado cuando se analizan las muestras tomadas en banda, mientras que el porcentaje de azufre y volátiles presenta un mejor comportamiento para las muestras recolectadas en la pila.

Sin embargo, siendo el poder calorífico la propiedad de mayor interés en este estudio, se considera relevante el hallazgo de este experimento inicial y se inicia un análisis de muestras suficientemente amplio para determinar si el comportamiento es constante, dado que es justamente este resultado el que contradice la hipótesis inicial de la investigación, relacionada con la disminución del poder calorífico obtenido en el proceso de generación con respecto al valor medido en las muestras recolectadas durante el proceso de construcción de la pila.

Los resultados reportados en la tabla I muestran diferencias porcentuales y además positivas entre las propiedades medidas en las muestras tomadas durante el proceso de consumo y las muestras recolectadas en la pila. Dado la cantidad reducida de muestras con las que se cuenta para esta prueba, se concluye que: se observa variación entre el poder calorífico, el porcentaje de ceniza y el porcentaje de humedad, que indica que el carbón muestreado en la banda posee mejores propiedades que el que se muestrea en la pila, en cualquiera de las bases en las que son reportados los resultados como se muestra en la Fig. 1, la Fig. 2 y la Fig. 3.

Estos resultados se relacionan con el tamaño del carbón que se analiza, dado que si se consideran carbones finos, el poder calorífico es diferente que cuando se miden tamaños de carbón mayores [2]. Esta segmentación de tamaños se genera debido al proceso de trituración que sufre el carbón antes de ser muestreado por medio del sistema mecánico instalado en la banda que lo transporta. 
TABla I. RESUltados ObTENidos DEL EXPERIMENTo D1.1

\begin{tabular}{|c|c|c|c|c|c|c|c|}
\hline \multicolumn{8}{|c|}{ Reporte Muestras Experimento D1.1 } \\
\hline \multicolumn{4}{|c|}{ D1.1 CONSUMO } & \multicolumn{4}{|c|}{ D1.1 PILA } \\
\hline PARÁMETRO & $\begin{array}{c}\text { AS } \\
\text { DET. }\end{array}$ & $\begin{array}{c}\text { DRY } \\
\text { BASIS }\end{array}$ & $\begin{array}{c}\mathrm{AS} \\
\mathrm{RCV}\end{array}$ & PRÁMETRO & $\begin{array}{c}\text { AS } \\
\text { DET. }\end{array}$ & $\begin{array}{c}\text { DRY } \\
\text { BASIS }\end{array}$ & $\begin{array}{c}\mathrm{AS} \\
\mathrm{RCV}\end{array}$ \\
\hline H. SUPERFICIAL & 434 & & & H. SUPERFICIAL & 499 & & \\
\hline PROMEDIO H.S & 434 & & & PROMEDIO H.S & 499 & & \\
\hline H. RESIDUAL & 221 & & & H. RESIDUAL & 226 & & \\
\hline PROMEDIO H.R & 221 & & & PROMEDIO H.R & 226 & & \\
\hline HUMEDAD TOTAL & & & 645 & HUMEDAD TOTAL & & & 714 \\
\hline CENIZAS & 1302 & & & CENIZAS & 1443 & & \\
\hline PROMEDIO CENIZAS & 1302 & 13,31 & 12,45 & PROMEDIO CENIZAS & 1443 & 1476 & 1371 \\
\hline VOLÁTILES & 3762 & & & VOLÁTILES & 371 & & \\
\hline PROM. VOLATILES & 3762 & 38,47 & 35,99 & PROM. VOLATILES & 371 & 3796 & 3525 \\
\hline AZUFRE & 133 & & & AZUFRE & 129 & & \\
\hline PROM. AZUFRE & 133 & 1,36 & 1,27 & PROM. AZUFRE & 129 & 132 & 123 \\
\hline PODER CALORÍFICO & 696100 & & & PODER CALORÍFICO & 677600 & & \\
\hline PROM.PODER CALORÍFICO & 696100 & 711800 & 665900 & PROM. PODER CALORÍFICO & 677600 & 693300 & 643800 \\
\hline HIDRÓGENO & & & 481 & HIDRÓGENO & & & 466 \\
\hline PODERCALORÍFICO NETO & & & 641200 & PODER CALORÍFICO NETO & & & 619800 \\
\hline
\end{tabular}

Fuente: Central Termoeléctrica de Sochagotá.

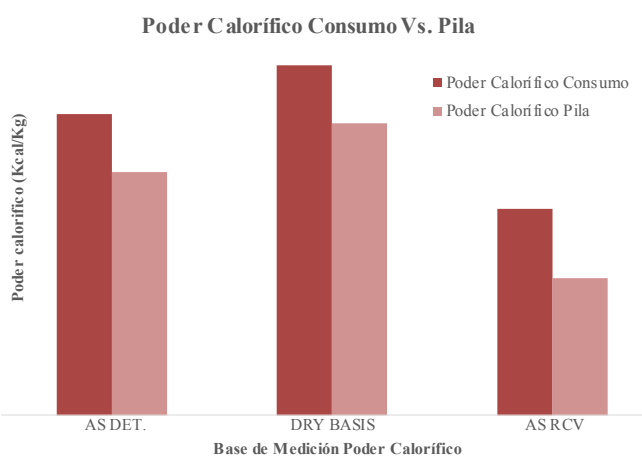

Fig. 1. Variación entre el Poder Calorífico medido en el consumo y en la pila en las diferentes bases de reporte de resultados. Fuente: Autores.

Porcentaje de Ceniza Consumo Vs. Pila

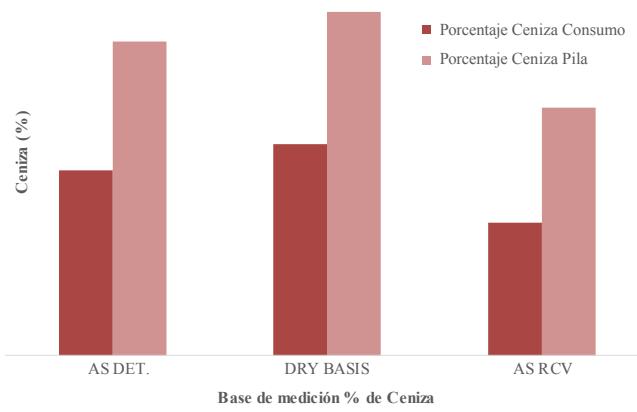

Fig. 2. Variación entre el Porcentaje de Ceniza medido en el consumo y en la pila en las diferentes bases de reporte de resultados. Fuente: Autores.

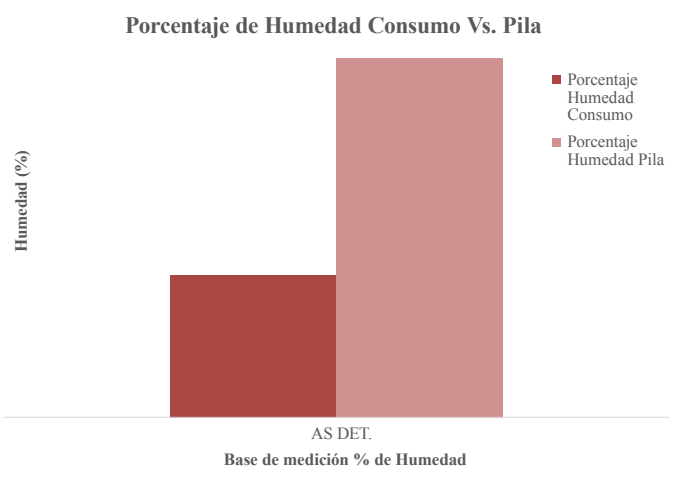

Fig. 3. Variación entre el Porcentaje de Humedad medido en el consumo y en la pila en las diferentes bases de reporte de resultados. Fuente: Autores.

\section{B. Experimento D 1.2 plan de muestreo preliminar}

Análisis pila de 45.000 toneladas y pruebas de compactación.

Descripción. La segunda prueba tiene como objetivo identificar los cambios en las propiedades medidas para las muestras de carbón recolectadas en el patio a diferentes niveles de compactación, exposición al ambiente y envejecimiento temporal. Para esta prueba se hace uso de una pila construida en la operación diaria de la Central Termoeléctrica de la cual no se conocen sus condiciones de apilamiento, dado que estaba construida cuando se inició esta investigación. 
Plan de consumo y muestreo. Dada la cantidad de carbón almacenada en esta pila (cerca de 45.000 t. métricas) se hace una sectorización teórica de la misma Fig. 2 para analizar el efecto de las condiciones ambientales sobre las propiedades del material, de acuerdo a la posición de donde es extraída la muestra, es decir en la que fue almacenada durante la construcción y consumo. Las posiciones evaluadas son definidas como compactación Alta, Media y Baja y el plan de muestreo dentro de la pila consiste en retirar el carbón de cada nivel de compactación y separarlo. Dada la sección transversal de la pila en la que se almacena el carbón y el efecto del ambiente sobre el área de la pila con mayor exposición se propone tomar las muestras teniendo una pared lo más vertical posible $\left(80^{\circ}\right.$ aprox.), para que los resultados no se vean afectados por las condiciones de temperatura y viento que afectan la superficie de la pila.

Luego de hacer la recolección de las muestras en la pila, el carbón es suministrado en el sistema de alimentación de la caldera donde se hace la recolección de muestras de consumo que son llevadas al laboratorio para ser preparadas y analizadas.

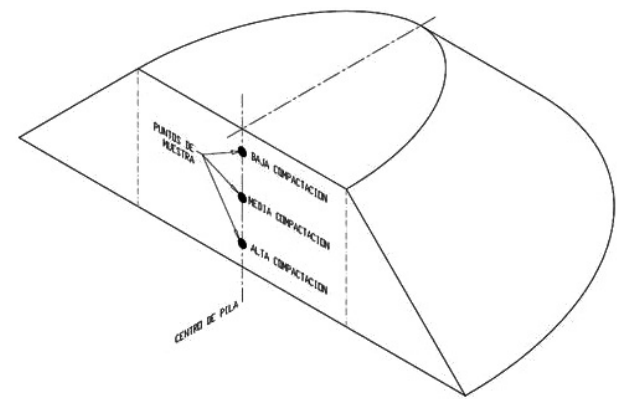

Figura 2. Consideraciones espaciales para la toma de muestras y el consuno de la pila. Fuente: Autores.

Presentación de resultados. El análisis de los datos obtenidos de esta pila de carbón se divide en diferentes escenarios dado el tamaño de la pila (aproximadamente 7 metros de altura en la parte central) y la información que se extrae de las propiedades del mismo durante los diferentes procesos dentro de la Central Termoeléctrica: se recopila información de las propiedades del material durante la construcción de la pila, de acuerdo a las condiciones propuestas para esta investigación y finalmente durante el consumo.

Resultados de construcción, datos recolectados durante la consolidación de la pila. En primera instancia se tomó la información de construcción de la pila para hacer un análisis de relación entre las siguientes propiedades:

- Poder Calorífico

- Porcentaje de Material volátil

- Porcentaje de Azufre

- Porcentaje de Humedad

- Porcentaje de Ceniza
Utilizando un análisis de regresión múltiple se obtiene que las propiedades que tienen correlación con el poder calorífico son: el porcentaje de humedad y el porcentaje de ceniza. La Fig. 4 representa el diagrama de correlación entre las propiedades del carbón, donde se puede evidenciar el comportamiento de cada una de las combinaciones de propiedades.

Correlación entre las propiedades del carbón medidas en la pila

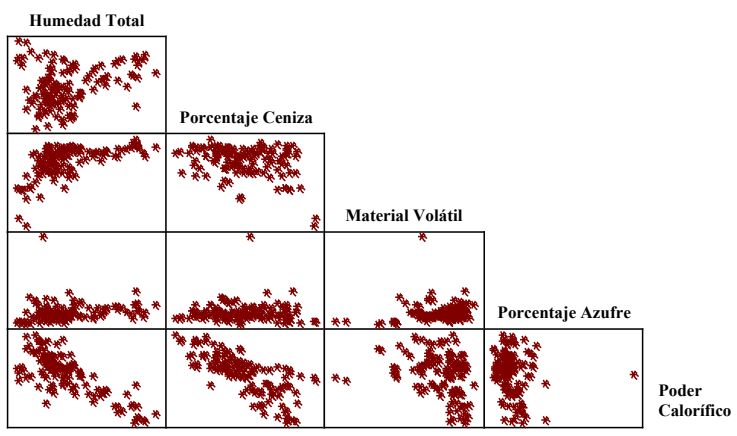

Fig. 4. Matriz de correlación de las propiedades del carbón medidas en la pila de almacenamiento. Fuente: Autores.

La relación lineal entre el porcentaje de ceniza, el porcentaje de humedad y el poder calorífico se analiza a partir de los valores estadísticos $\mathrm{P}$ valor $(<0,05)$ y correlación de Pearson $(>0,07)$ mostrados en la tabla II, estos valores indican que dicha relación es significativa y por lo tanto el poder calorífico se ve afectado ante el cambio en el contenido de humedad y ceniza del carbón. Para las demás propiedades se encuentra un valor $\mathrm{P}$ mayor a 0,05 y en los casos donde este estadístico es menor, el coeficiente de correlación de Pearson es menor que $70 \%$, de tal modo que las propiedades sobre las que se enfatiza el análisis son la humedad y la ceniza.

Resultados de consumo, datos recolectados para las muestras tomadas por el sistema mecánico de muestreo en la banda transportadora que alimenta la caldera. Los resultados de las muestras recolectadas durante el consumo de las 45.000 t otorgan información sobre las propiedades del carbón mencionadas en el análisis anterior. Para la revisión de información se cuenta con un total de 50 informes de laboratorio donde inicialmente se busca definir gráfica y estadísticamente el comportamiento de las propiedades frente al poder calorífico que permita establecer una comparación con los hallazgos definidos con la información recolectada durante la construcción.

En la matriz de la fig. 5 se evidencia la correlación entre las combinaciones de variables, considerando la misma escala del gráfico de la información correspondiente a la construcción, con el objetivo de hacer de estas ilustraciones puntos de referencia comparables entre sí. 
Tabla II. Análisis de correlación entre propiedades del carbón vs. Poder Calorífico medidos en Pila

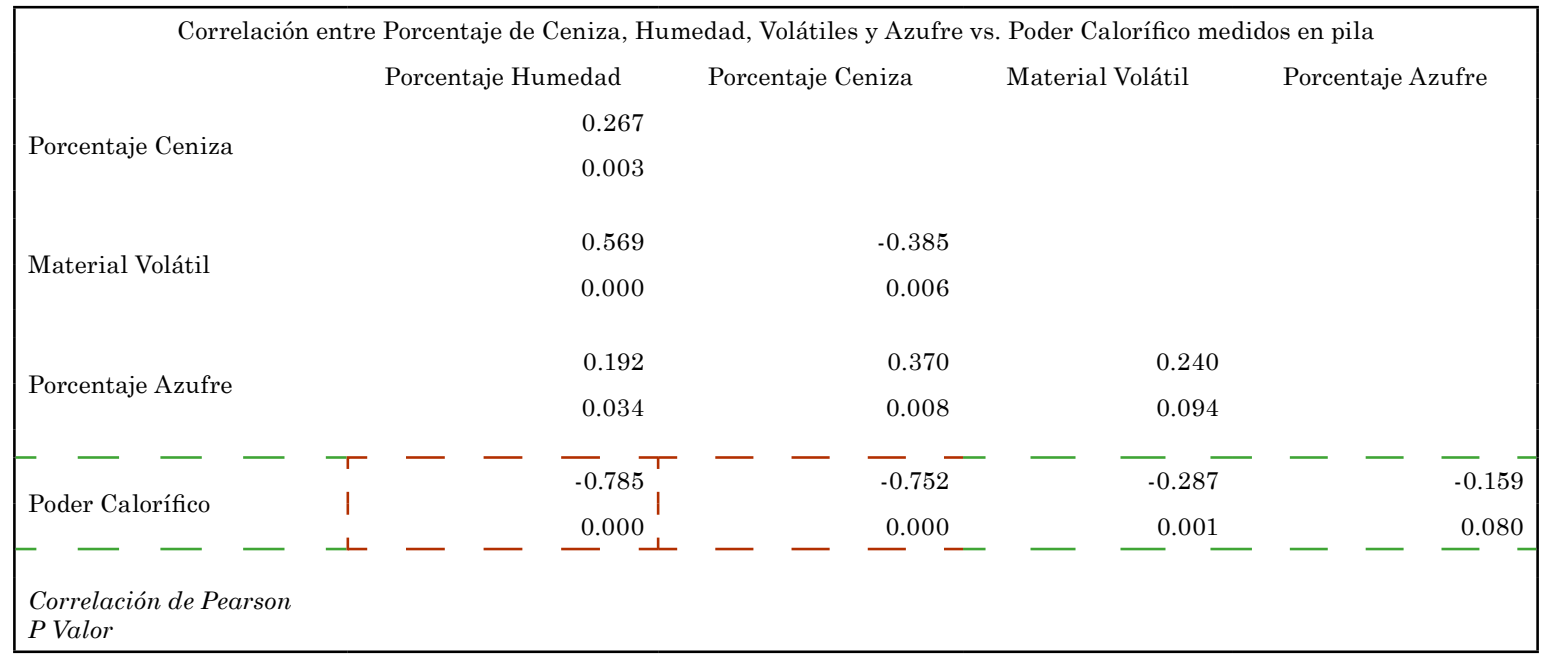

Fuente: Autores.

Correlación de las propiedades del carbón medidas en el consumo

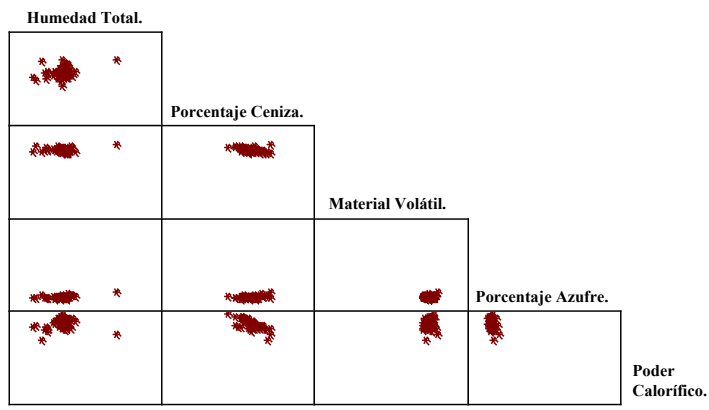

Fig. 5. Matriz de correlación de las propiedades del carbón medidas en el consumo.

Fuente: Autores.
Los datos recolectados durante el consumo están agrupados en rangos de variación menor para todas las propiedades medidas, con respecto a los rangos de la información obtenida para las muestras de la construcción, bajo la salvedad que se tiene un menor número de muestras en esta etapa del proceso por la frecuencia con que se tomaron y analizaron las muestras.

Como se evidencia en la tabla III y bajo las condiciones de análisis planteadas para las muestras tomadas en la pila, se observa según la matriz de correlación y los estadísticos de prueba que la única propiedad que presenta relación con el poder calorífico es el porcentaje de ceniza. A diferencia del análisis de los datos de la pila no se observa relación entre el porcentaje de humedad y el poder calorífico medido.

Tabla III. Análisis de correlación entre propiedades del carbón vs. Poder Calorífico medidos en el consumo.

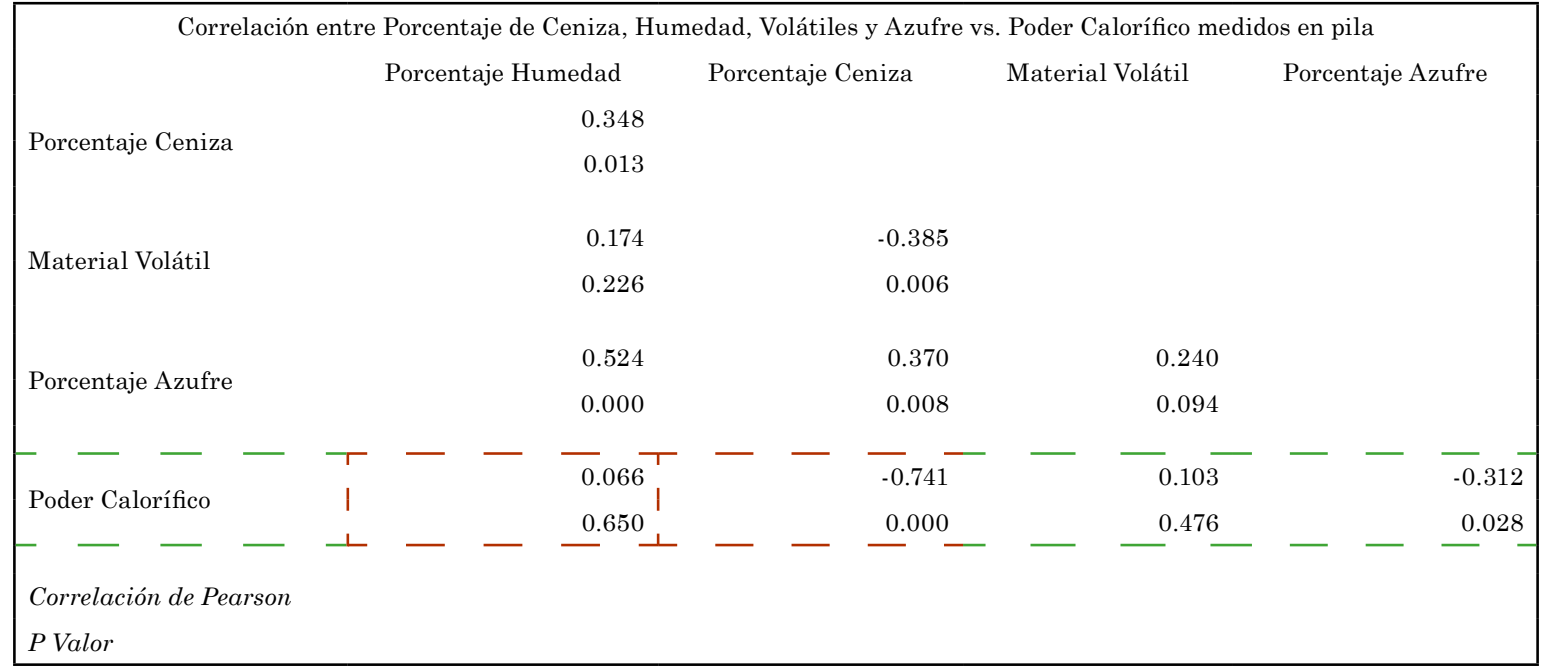


Comparación de resultados de construcción y resultados de consumo. Una vez identificado el comportamiento independiente de las muestras tomadas durante la construcción y durante el consumo, es necesario establecer una comparación para definir el estado de los resultados de las pruebas frente a la hipótesis inicial de la investigación (el poder calorífico medido en la pila es mejor que el poder calorífico medido en la banda).

Para revisar esta afirmación frente a los datos recolectados se elabora un histograma para dos poblaciones que permite visualizar el comportamiento de la media y la variación de cada una de las poblaciones (Fig. 6)

Histograma: Poder Calorífico vs. Poder Caloriífico Consumo

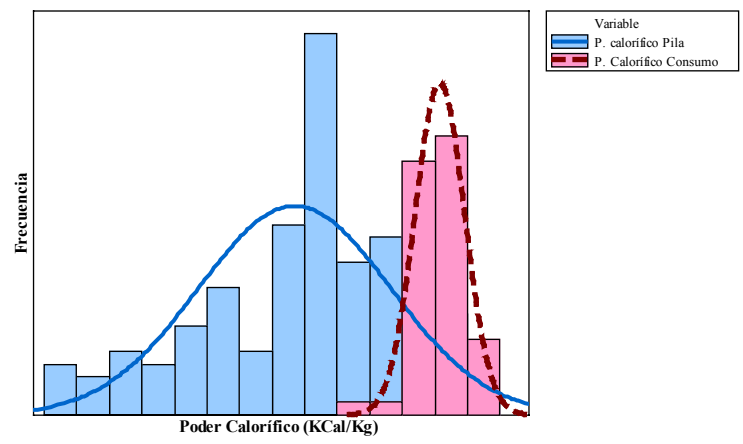

Fig. 6. Histograma de Poder Calorífico Pila Vs Poder Calorífico Consumo para 45.000 t de carbón. Fuente: Autores.

En promedio para los datos de consumo se obtiene un poder calorífico de $716.500 \mathrm{Kcal} / \mathrm{Kg}$ con una desviación estándar de $7.641 \mathrm{Kcal} / \mathrm{Kg}$, mientras que para los datos de la pila se tiene un poder calorífico promedio de $671.900 \mathrm{Kcal} / \mathrm{Kg}$ con una desviación estándar de 295.700. De esta información se infiere que el desempeño (poder calorífico) es mejor en las mediciones realizadas durante el consumo que en el registro de la construcción, haciendo la comparación de los valores promedios y la desviación estándar, lo cual contradice la hipótesis inicial objeto de investigación.

Es necesario resaltar que se tiene variación en el tamaño de la muestra de las dos poblaciones, siendo 122 datos para la construcción y 50 para el consumo; sin embargo, resulta inquietante el comportamiento de los datos de consumo, ya que aunque están representados por un número de datos inferior son un muestreo representativo de la pila de carbón pero el comportamiento evidenciado en la Fig. 6 muestra dos poblaciones diferentes con media y desviación estándar particulares.

Resultados de factores propuestos (compactación, exposición y envejecimiento). Finalmente, se desea encontrar la relación del poder calorífico con factores definidos de forma estratégica, que se consideraron como posibles incidentes en las mediciones realizadas. Para esto durante el almacenamiento y previo al consumo se tomaron incrementos en la pila que fueron analizados según su localización y tiempo de exposición.

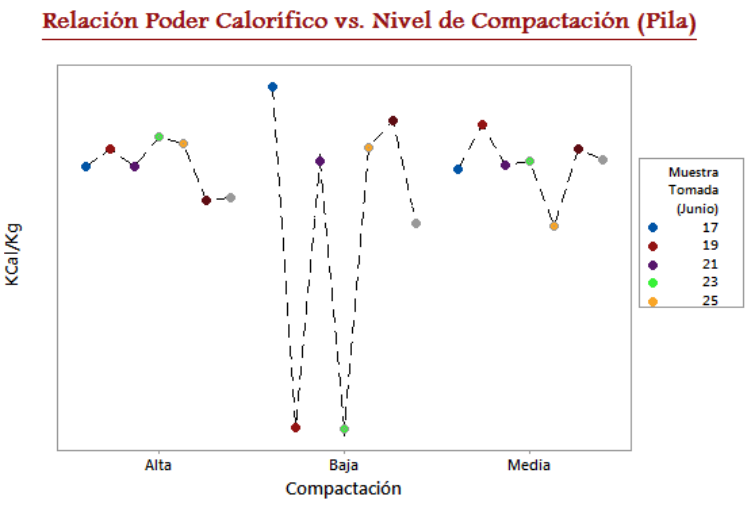

Fig. 7. Relación entre la compactación del carbón en la pila y el poder calorífico medido Fuente: Autores.

Según la zona de consumo del carbón dentro de la pila y los días transcurridos desde el inicio del experimento se logra observar que no existe una relación definida entre los factores compactación, envejecimiento y exposición al ambiente con el poder calorífico. Sin embargo, es de resaltar que el efecto por envejecimiento no se pudo definir con claridad debido a falta de información en cuanto a la secuencia de construcción de la pila.

El poder calorífico de mayor valor se obtuvo en condiciones de compactación baja, alta exposición al ambiente y primera fecha de consumo. En compactación baja también se encontraron los datos con el más bajo desempeño (poder calorífico) y la mayor variación en las mediciones.

\section{Experimento D 1.2 plan de muestreo preliminar}

\section{Construcción Pila de 1500 t de carbón proveniente de un único proveedor.}

Descripción. Con el objetivo de identificar la incidencia de las condiciones ambientales (exposición al ambiente) y tiempo de almacenamiento (envejecimiento) del carbón en el patio, se construyó una pila de carbón con 1500 t de material proveniente de un único proveedor de la Central Termoeléctrica lo que permitió eliminar el efecto de la mezcla de carbón de diferentes proveedores sobre las variación presentada en la medición de propiedades. Dado que la construcción se realizó en pocos días se podría esperar que su origen fuera de una única mina y/o veta, esto sin embargo no se pudo comprobar.

Construcción, plan de consumo y muestreo. La construcción de la pila tuvo una duración de 8 días donde se muestreó cada camión con el fin de conocer las propiedades del material recibido, posteriormente esta pila fue almacenada durante 20 días bajo condiciones ambientales conocidas y se inició el 
plan de consumo parcial (300 t cada 15 días), permitiendo que una porción del material de la pila permaneciera almacenado durante períodos de tiempo controlados.

Para cada fecha de consumo se recolectó una muestra de las 300 t retiradas de la pila, adicionalmente esta cantidad de carbón fue dividida en porciones de 100 t a cada una de las cuales se les tomó una muestra con el fin de comparar los resultados obtenidos en las muestras de $100 \mathrm{t}$ contra los resultados de la muestra general y de esta forma evaluar la representatividad de la misma. Cuando se finaliza la toma de muestras en la pila el carbón es enviado a la línea de alimentación una vez las tolvas y la banda han sido despejadas para garantizar que las muestras recolectadas durante el consumo no presentan mezclas de material que puedan sesgar los resultados a evaluar. Allí se programa el sistema mecánico de muestreo para tomar incrementos de las primeras 100 t que suben por la banda, este procedimiento se repite cada 100 t hasta completar el proceso. Para obtener la muestra general del proceso de consumo y poder evaluar el efecto de los promedios en los resultados medidos se mezclan 2 kilos de cada una de las tres bolsas tomadas en la banda para formar la muestra general de las $300 \mathrm{t}$.

La recolección inmediata de las muestras en los dos escenarios se usó para descartar la variación encontrada entre las propiedades medidas en la pila y las propiedades medidas previo al consumo, por el efecto del tiempo transcurrido entre la toma de muestras en cada etapa. El procedimiento descrito anteriormente para la recolección de muestras en la pila y durante el consumo fue ejecutado para cada 300 t en 5 fechas programadas.

Presentación de resultados. Los resultados obtenidos en este experimento se presentan divididos en dos grupos: los de la pila y los del consumo. Esta información se divide en cuatro tablas correspondientes a cada una de las fechas programadas para la ejecución de la prueba. Las pruebas denominadas como 1 (tomada el 12 de Septiembre), 2 (tomada el 26 de Septiembre), 3 (tomada el 10 de Octubre) y 5 (tomada el 19 de Noviembre), se analizan en las Fig. 8 a Fig. 15, donde se excluye la prueba número 4 que no fue posible realizar.

Resultados de construcción. Las muestras recolectadas en los camiones se almacenan hasta completar $500 \mathrm{t}$ de material recibido, posteriormente las muestras tomadas en los camiones que conformaron esta cantidad son mezcladas para obtener una sola muestra. En los resultados suministrados se encuentran 4 mediciones correspondientes a 47 camiones y cada una de estas representa una cantidad de carbón diferente a las $500 \mathrm{t}$ del procedimiento establecido, por esta razón y la cantidad reducida de información los resultados obtenidos para estas muestras no permiten realizar ningún tipo de análisis.

Comparación resultados obtenidos en la pila y en el consumo. De esta forma el primer análisis realizado corresponde a una comparación entre los resultados de poder calorífico y el porcentaje de ceniza obtenidos en las muestras recolectadas en la pila y las muestras recolectadas en el consumo, esta comparación devela una variación significativa entre los valores de poder calorífico medidos en los dos escenarios, a pesar de ser una muestra selecta de material y haber tenido un tratamiento especial durante la recolección. La diferencia entre el poder calorífico medido en el consumo y en la pila es positiva, lo que nuevamente contradice la hipótesis inicial con la que se inició la investigación.

A continuación se presentan la comparación del poder calorífico medido en la pila y en los consumos correspondientes a las pruebas programadas cada 15 días, las Fig. 8 y Fig. 9 representan una correlación entre el porcentaje de ceniza y el poder calorífico medido para los dos escenarios de medición en la primera prueba programada. Así mismo las Fig. 10 y 11 representan los resultados de la prueba número 2.

\section{Prueba 1}

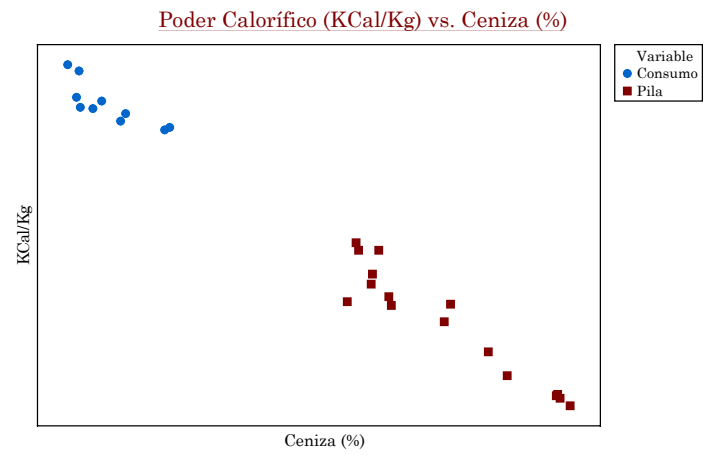

Fig. 8. Diagrama de correlación poder calorífico vs. ceniza. Fuente: Autores.

Tabla IV. Propiedades medidas en las muestras recolectadas en los Camiones.

\begin{tabular}{|c|c|c|c|c|c|}
\hline $\begin{array}{c}\text { Peso Representado } \\
\text { Kg }\end{array}$ & $\begin{array}{c}\text { Humedad } \\
\text { Total \% }\end{array}$ & Cenizas \% & $\begin{array}{c}\text { Materia } \\
\text { Volátil \% }\end{array}$ & Azufre \% & $\begin{array}{c}\text { Poder Calorífico Neto } \\
\text { Kcal/Kg }\end{array}$ \\
\hline 376.000 & 619 & 1957,14743 & 3529,47447 & 106,598444 & $633.621,1491$ \\
\hline 532.000 & 537 & 1275,49403 & 3848,67378 & 126,80968 & $694.923,3858$ \\
\hline 198.000 & 531 & 1812,22938 & 3704,72067 & 117,224628 & $647.615,3765$ \\
\hline 526.000 & 525 & 1434,30079 & 3767,81003 & 116,094987 & $678.738,7863$ \\
\hline
\end{tabular}

Fuente: Autores. 


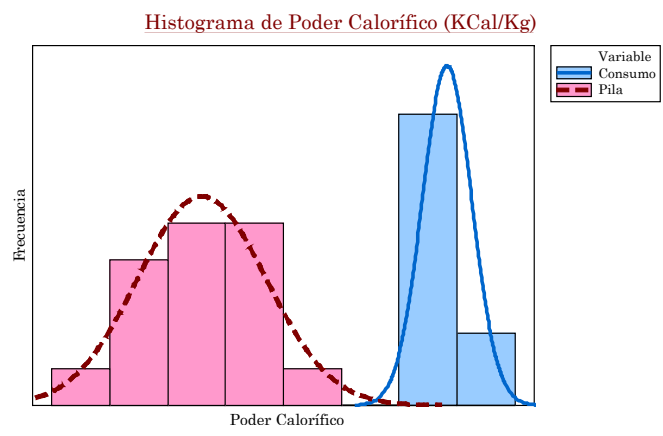

Fig. 9. Histograma de Poder Calorífico prueba 1. Fuente: Autores.

Prueba 2

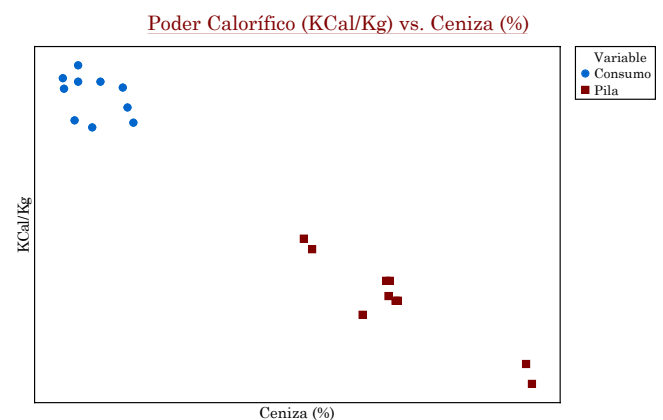

Fig. 10. Diagrama de correlación poder calorífico vs. ceniza. Fuente: Autores.

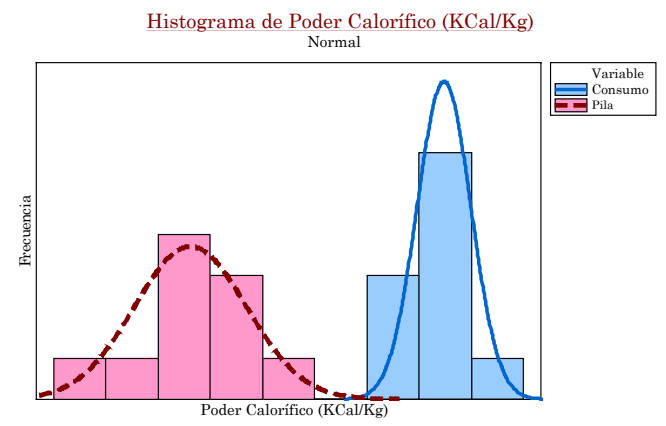

Fig. 11.Histograma de Poder Calorífico prueba 2. Fuente: Autores.

Prueba 3

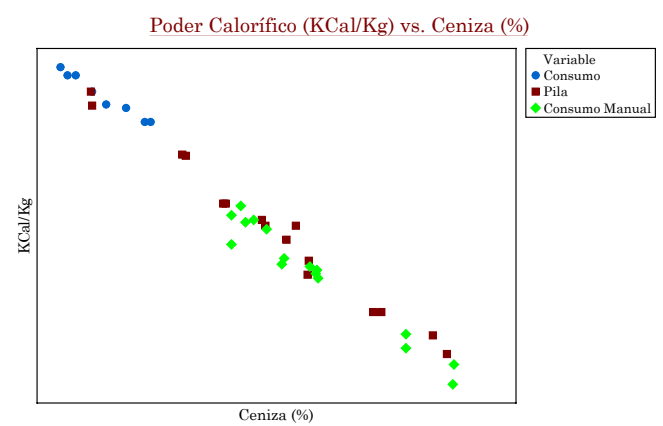

Fig. 12. Diagrama de correlación poder calorífico vs. ceniza. Fuente: Autores.

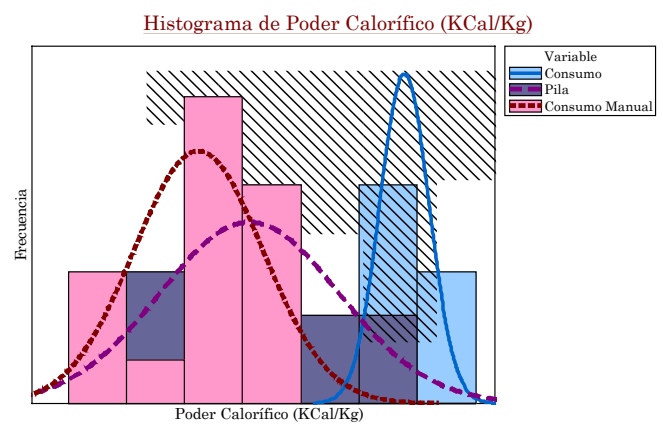

Fig. 13. Histograma de Poder Calorífico prueba 3. Fuente: Autores.

Prueba 5

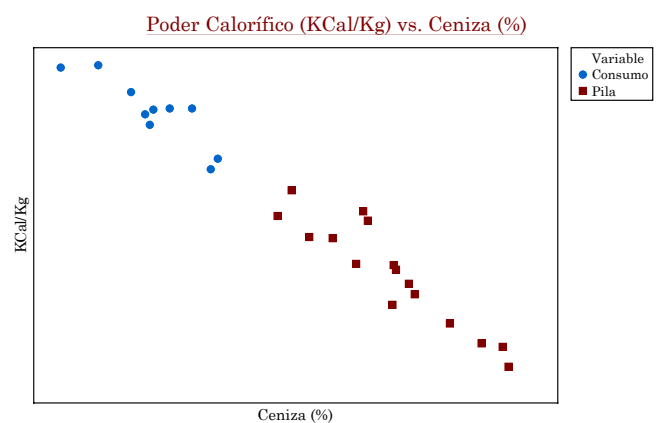

Fig. 14. Diagrama de correlación poder calorífico vs. ceniza. Fuente: Autores.

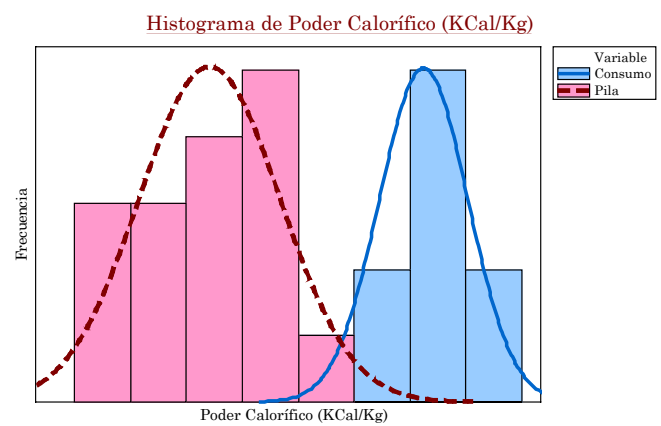

Fig. 15. Histograma de poder calorífico prueba 5. Fuente: Autores.

Dados el control sobre la consecución y el control en los procedimientos realizados con las 1500 t de carbón, se esperaría que este análisis indique valores confiables para determinar la variación existente entre las propiedades medidas en el camión, la pila y el consumo. Sin embargo, las gráficas muestran el comportamiento del poder calorífico cuando es medido en la pila y en la banda como dos conjuntos de datos segmentados sin ninguna relación, lo que genera preocupación cuando se tiene una muestra que teóricamente es la misma y no existe un factor evidente que afecte el valor del poder calorífico, del porcentaje de humedad y del porcentaje de ceniza. 
Es de especial interés la información que muestra la Fig. 12 y la Fig. 13, donde se hace la comparación para la "Prueba 3" de los valores obtenidos en la pila y durante el consumo, puesto que por iniciativa de la Central Termoeléctrica para esta prueba se generaron muestras durante el consumo utilizando otra metodología, que consistió en operar el tomador de muestras de la línea de alimentación de forma manual y no programada como normalmente se lleva a cabo. Finalmente la prueba 5 permite observar segregación de los datos como lo muestran la 14 y 15 que corresponden a la correlación de ceniza y poder calorífico y a un histograma del poder calorífico respectivamente.

La evaluación del efecto de las condiciones ambientales sobre el comportamiento de las propiedades se determina en las Fig. 16 a Fig. 21, en las cuales se comparan los valores obtenidos para las propiedades de poder calorífico, ceniza $\mathrm{y}$ humedad en cada una de las pruebas hechas para la pila y el consumo respectivamente. En estas gráficas se muestra que el efecto del clima es evidente cuando se compara el porcentaje de humedad en cada una de las pruebas.

\section{Porcentaje de humedad}

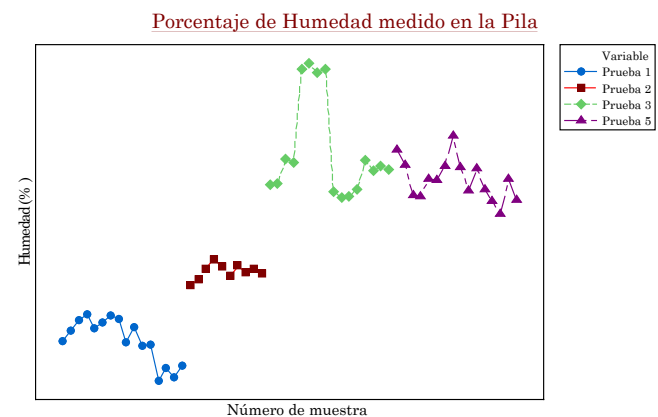

Fig. 16. Porcentaje de Humedad medido en la pila. Fuente: Autores.

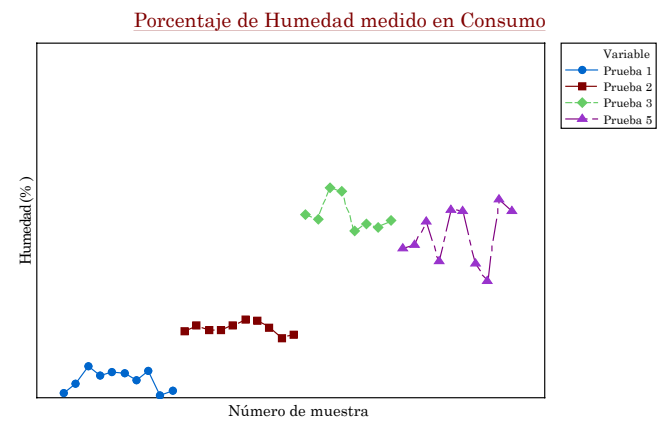

Fig. 17. Porcentaje de Humedad medido en el consumo.

Fuente: Autores.

\section{Porcentaje de ceniza}

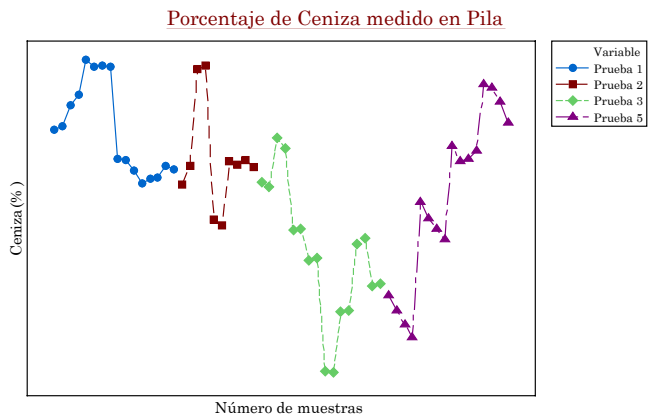

Fig. 18. Porcentaje de Ceniza medido en Pila. Fuente: Autores.

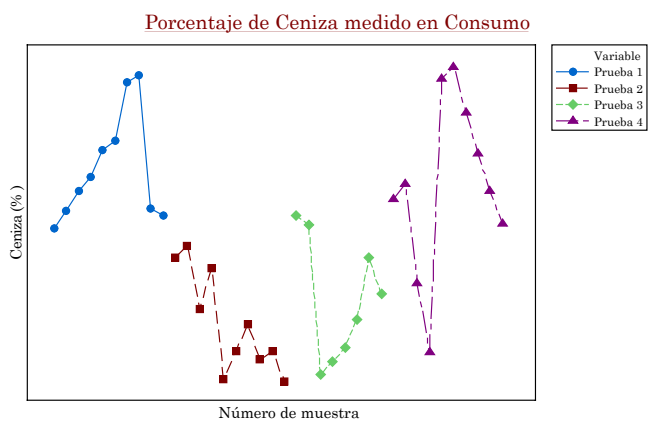

Fig. 19. Porcentaje de Ceniza medido en consumo. Fuente: Autores.

\section{Poder calorifico}

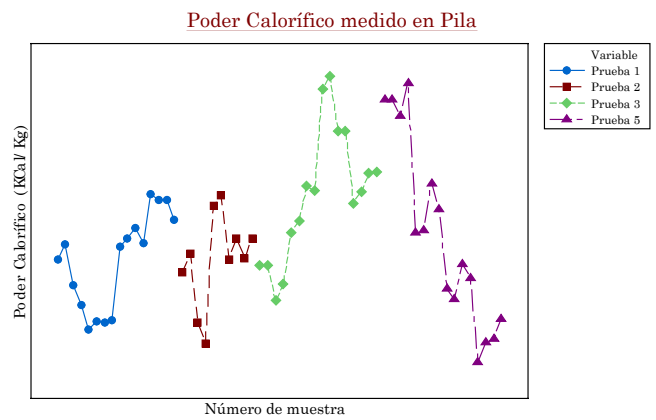

Fig. 20. Poder Calorífico medido en Pila. Fuente: Autores.

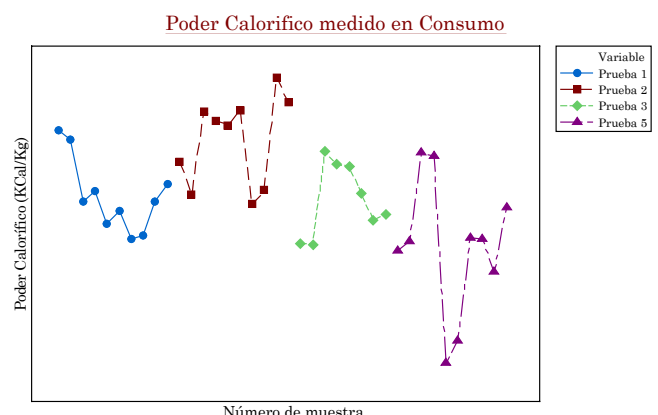

Fig. 21. Poder Calorífico medido en consumo. Fuente: Autores. 
El comportamiento de las propiedades medidas cuando el envejecimiento del material estudiado aumenta, no es claro por lo cual se muestran los valores promedio de lluvia registrados durante el almacenamiento del carbón que fue consumido en cada una de las pruebas.

En la Tabla V se evidencia que el promedio de lluvias presentó variaciones considerables durante el tiempo de envejecimiento para las pruebas 2 , 3 y 5 , lo que responde al comportamiento de la humedad cuando se comparan las cuatro pruebas realizadas en la pila y durante el proceso de consumo. Así mismo los valores de temperatura en el periodo de envejecimiento de cada una de las pruebas no muestran variaciones de consideración que tampoco se evidencian en las gráficas. Debido a la correlación existente entre la humedad y el poder calorífico se encuentra que el poder calorífico disminuye en algunas mediciones hechas al material que fue expuesto a un envejecimiento mayor, aunque las tendencias no sean consistentes para concluir que existen perdidas en el poder calorífico dado los aumentos presentados en la humedad.

Finalmente cuando se comparan los resultados del Poder Calorífico medido en cada una de las pruebas, tanto en la pila como en el consumo frente al poder calorífico observado en las cuatro muestras que se recolectaron y midieron durante la recepción del carbón se encuentra que estos cuatro datos abarcan el rango en el que se mueven los resultados de las muestras medidas en los dos escenarios. En la Fig. 22 se muestra la correlación entre el porcentaje de ceniza y el poder calorífico para los resultados de las pruebas 1 y dos frente a los resultados obtenidos en las muestras del camión.

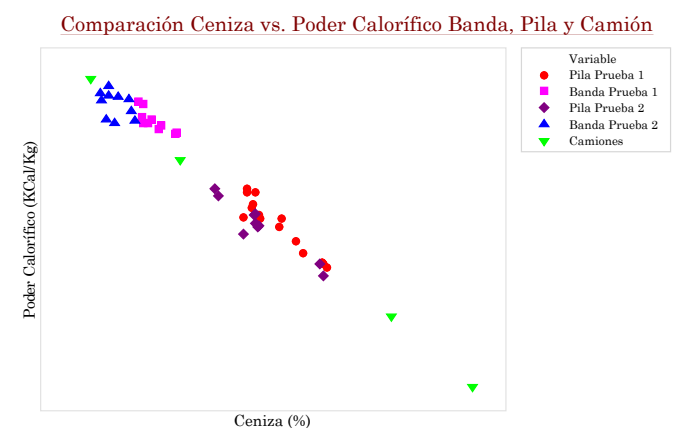

Fig. 22. Comparación porcentaje de ceniza vs. poder calorífico pila, banda y camión. Fuente: Autores.

\section{Conclusiones}

En el análisis y evaluación de los resultados de las mediciones es importante tener un contexto de los diferentes métodos de presentación de resultados que sin duda alguna generan diferencias entre la información obtenida. En el caso del porcentaje de ceniza es importante conocer que la influencia de esta característica sobre el poder calorífico se presenta de forma clara cuando el material es analizado discriminando el porcentaje de humedad (Base Seca). De esta manera, para un estudio del carbón es de vital importancia establecer el mismo criterio de análisis ya que de no ser así se registran resultados sin ningún tipo de relación y que llevan a conclusiones equivocas.

Durante el proceso de recolección de información, que incluye las etapas de muestreo en la recepción, en la pila de almacenamiento y finalmente durante el consumo, existe una situación en el muestreo que indica una brecha entre los valores de poder calorífico medidos en la pila y en el consumo, contraria a la situación inicial planteada por la Central Termoeléctrica. Sin embargo en la Fig. 22 se muestra la correlación entre el porcentaje de ceniza y los valores de poder calorífico medidos en la recepción (camión), pila y consumo y se observa que las muestras del camión abarcan las mediciones de los otros dos escenarios de medición.

Los resultados encontrados en las tres pruebas planteadas y realizadas muestran el mismo comportamiento, cuando se hace la comparación entre las muestras de la pila y del consumo. Adicionalmente la relación del poder calorífico medido en la pila y durante el consumo es nula ya que los resultados se agrupan como dos segmentos de datos, aun siendo de la misma población (ver Fig. 8 a Fig. 12).

Adicionalmente es recomendable establecer procedimientos estándar para llevar a cabo los procesos de medición del carbón, debido a la aleatoriedad de los resultados medidos y posteriormente analizados. A lo largo del análisis de la información obtenida sobre el carbón se encuentra que una de las causas de esta divergencia es la naturaleza del material, el cual puede poseer diferentes características a pesar de provenir incluso de una misma mina y de una misma veta de carbón.

Tabla V. Condiciones de lluvia y temperatura Registradas durante los PERiodos de EnVEjecimiento.

\begin{tabular}{|l|l|l|l|}
\hline \multicolumn{1}{|c|}{ Periodo de tiempo medido* } & Promedio de lluvias (mm) $^{*}$ & Promedio Temperatura $^{\circ} \mathbf{C}$ & \multicolumn{1}{|c|}{ Prueba afectada $^{*}$} \\
\hline $09 / 09 / 2014-12 / 09 / 2014$ & 0 & 12,560241 & Prueba 1 \\
\hline $09 / 09 / 2014-26 / 09 / 2014$ & 0,11729622 & 12,7912525 & Prueba 2 \\
\hline $09 / 09 / 2014-10 / 10 / 2014$ & 0,16090584 & 12,9845054 & Prueba 3 \\
\hline $09 / 09 / 2014-19 / 11 / 2014$ & 0,17676487 & 13,2095609 & Prueba 5 \\
\hline
\end{tabular}

* Los registros de lluvia fueron suministrados por la Central Termoeléctrica objeto de estudio).

* Los datos de la tabla representan los registros a partir del 09/09/2014 dada la información suministrada.

Fuente: Autores. 
Lo que implica considerar una clasificación en la recepción y posterior almacenamiento de acuerdo a los porcentajes de ceniza obtenidos en la recepción.

Los procedimientos planteados para llevar a cabo el análisis experimental pretendían establecer condiciones específicas que permitieran hacer un análisis de relación entre muchas variables ante resultados no esperados, para evaluar las posibles causas de dichos comportamientos en el carbón. Al no llevar a cabo todos los procedimientos solicitados desde el inicio, fue imposible obtener un análisis relacional robusto entre los parámetros del proceso (DOE).

Finalmente se encuentra como la causa principal de la diferencia entre los valores de las propiedades medidas, especialmente del poder calorífico, en las muestras de la pila y del consumo se debe al funcionamiento del mecanismo de medición, considerado seguro desde el planteamiento del problema. Esta diferencia se debió a un desgaste en la pieza del sistema mecánico de muestreo no detectado (el desgaste del barredor solo removía material fino de la banda). Esto empezó a ser notorio, aunque no comprendido, desde los resultados del primer experimento.

\section{Bibliografía}

[1] ASTM D 409,1983," Standard Test Method for Grindability of Coal by the Hardgrove-Machine Method", ASTM International, West Conshohocken, PA, 1983, www.astm. org.

[2] J. Ekmann and P. LE. "Coal Storage and Transportation”. Pennsylvania: U.S. Department of Energy Pittsburgh, pp. 551-579.

[3] ASTM E 152-72, 1979,"Standard recommended practice for choice of sample size to estimate the average quality of a lot or process", ASTM International, West Conshohocken, PA, 1979, www.astm.org.
[4] Air and Energy Engineering Research Laboratory. (1985). "Research and Development of Coal sampling and analysis: Methods and models", In EPA, Environmental Protection Agency. Considerations of measurement error (PP 107- 120).Washington DC, EE.UU.

[5] ASTM D 2234, 1976," Standard Practice for Collection of a Gross Sample of Coal", ASTM International, West Conshohocken, PA, 1976, www.astm.org.

[6] ASTM D 3176-84, 1984,"Standard Test Method for Ultimate Analysis of Coal and Coke", ASTM International, West Conshohocken, PA,1984, www.astm.org.

[7] L. P. Fattiand T. J. Stewart, 1986, "Quality Control in Export Coal". In The Journal of the Operational Research Society, Vol. 37, No. 11 Great Britain, 1986, pp.1073-1080.

[8] ASTM D 3174-82, 1982,"Ash in the Analysis Sample of Coal and Coke from Coal", ASTM International, West Conshohocken, PA, 1982, www.astm.org.

[9] ASTM D 3175- 82, 1982,"Volatil Matter in the Analysis Sample of Coal and Coke", ASTM International, West Conshohocken, PA, 1982, www.astm.org.

[10] ASTM D 3302- 07, 2007,"Standard Test Method for Total Moisture in Coal", ASTM International, West Conshohocken, PA, 2007, www.astm.org.

[11] ASTM D 9725-95, 2001," Standard Test Methods for Analysis of Coal and Coke Ash", ASTM International, West Conshohocken, PA, 2001, www.astm.org.

[12] ASTM Standard D 346, 1991."Standard Practice for Collection and Preparation of Coke Samples for Laboratory Analysis", ASTM International, West Conshohocken, PA, 1991, www.astm.org.

[13] ASTM D 3180-89, 1989,"Calculating Coal and Coke Analyses from As-Determined to different Bases", ASTM International, West Conshohocken, PA, 1989, www.astm.org.

[14] A. Requena, Ensayos de Caracterización del Carbón Mineral [en línea]. Venezuela: Universidad Simón Bolívar, 2012 Disponible en: http://es.slideshare.net/ alejandrorequena/ensayos-para-la-caracterizacin-delcarbon. 